\title{
L'approche belge pour mieux prévenir les risques psychosociaux en général et le harcèlement psychologique en particulier
}

\author{
Isabelle Hansez - BELGIQUE
}

Chargée de cours adjointe, Service de psychologie du travail - Faculté de psychologie et des sciences de l'éducation - Université de Liège - boulevard du Rectorat, 5 - 4000 Liège 1 Belgique - 3243662092 - ihansez@ulg.ac.be

Daniel Faulx - BELGIQUE

Chercheur-intervenant, Service de psychologie sociale des groupes et des organisations Université de Liège - boulevard du Rectorat, 5 - 4000 Liège 1 Belgique - 3243664675 Daniel.Faulx@ulg.ac.be

Aude Mahy - BELGIQUE

Conseiller en prévention aspects psychosociaux, Service externe pour la prévention et la protection au travail, Université de Liège - boulevard du Rectorat, 5 - 4000 Liège 1 Belgique 3242349763 - aude.mahy@cbmt.be

\section{Résumé}

La législation est un levier important de la mise en place de mesures de prévention des risques professionnels. La directive européenne n89/391/CEE donne l'impulsion à une législation commune au sein de l'Union en matière de prévention des risques professionnels et de protection de la sécurité et de la santé des travailleurs. Chaque pays membre a la responsabilité de transposer cette directive dans sa législation nationale. Aujourd'hui, tous les états membres ne sont pas arrivés au même stade maturation dans leur politique de prévention des risques psychosociaux. En Belgique, l'évolution de la législation en matière de santé et de sécurité au travail s'est caractérisée par un élargissement très marqué de la notion de sécurité au travail vers celle de la santé, puis de bien-être au travail et enfin plus spécifiquement de violence, harcèlement moral ou sexuel au travail. La loi sur le bien-être au travail de 1996 laisse apparaître pour la première fois la notion de prévention des risques psychosociaux. Une convention collective de travail (la CCT 72 de 1999) porte plus spécifiquement sur la prévention $\mathrm{du}$ stress professionnel. Plus récemment, la loi sur la protection contre la violence, le harcèlement moral ou sexuel au travail a été votée en 2002. Le domaine couvert par cette loi a été ajouté aux domaines qui font partie de la notion de bien-être dans la loi de 1996 sur le bienêtre des travailleurs. Après la description de ces législations, ce papier présente les pratiques en matière de recherche et d'interventions dans les entreprises qui découlent directement de ces nouvelles dispositions légales. Enfin le recul, certes encore minime, de leur application alimente la réflexion sur les répercussion de ces pratiques en entreprise ou auprès des travailleurs.

\section{Abstract}




\section{Introduction}

Au-delà des chiffres, l'importance du phénomène de mal-être au travail est très certainement liée à l'évolution du monde du travail (Sauter, 1995). En Belgique comme ailleurs, les entreprises sont forcées de se placer dans une perspective de compétitivité internationale. Les contraintes de rentabilité sont souvent très élevées au regard de la tension et de la flexibilité. Les contraintes de temps, notamment, ont fortement augmenté en Europe mais aussi ailleurs (Fondation européenne pour l'amélioration des conditions de vie et de travail, 1997). Le contexte du travail change rapidement : on évolue aujourd'hui vers un secteur tertiaire majoritaire, avec toutes les exigences que cela implique, par exemple sur le plan de la clientèle ou des contacts humains, du just in time management, etc. On évolue aussi vers une économie qui dépasse le cadre traditionnel des huit heures de travail par jour et s'étend tout naturellement à vingt-quatre heures, ce qui suppose des pratiques de flexibilité qui ne sont pas toujours bénéfiques au travailleur. La nature même de la force de travail a changé, ce qui pose de nouvelles problématiques liées notamment aux travailleurs âgés.

\section{La législation sur le bien-être au travail : la situation en Europe et le cas de la Belgique}

Les années 1990 ont été marquées par une préoccupation croissante du législateur pour les risques liés au travail, au niveau de l'Europe ${ }^{1}$ d'abord, puis au niveau des États membres de l'Union européenne. De manière générale, on constate un élargissement très marqué de la notion de sécurité au travail vers la notion de santé puis de bien-être au travail, ainsi qu'un ascendant de la prévention et de la gestion des risques sur la protection des travailleurs.

La Belgique est un bon exemple des sensibilités européennes à l'égard de l'apparition de la problématique des risques psychosociaux et du stress dans le monde du travail. Elle est dotée de fortes structures syndicales et d'une législation importante pour la protection du travailleur comprise dans le Code sur le bien-être au travail (1993), qui a remplacé le Règlement général pour la protection du travailleur datant de 1946. En matière de législation, elle figure parmi les premiers pays européens à promulguer une loi sur le bien-être des travailleurs dans l'exécution de leur travail. Cette loi s'accompagne d'arrêtés d'application royaux, dont les trois principaux datent du 27 mars 1998 ; ils concernent la politique du bien-être des travailleurs lors de l'exécution de leur travail, les services internes de prévention et les services externes de prévention (Ministère fédéral de l'Emploi et du Travail, 2000)².

Cette nouvelle législation repose essentiellement sur les aspects suivants :

- La définition du bien-être qui est lié à l'ensemble des facteurs concernant les conditions dans lesquelles le travail est effectué : la sécurité au travail, la protection de la santé du travailleur, la charge psychosociale, l'ergonomie, l'hygiène au travail, l'embellissement

1. Directive européenne (1989) : Directive-cadre n 89/391/CEE.

2. Loi du 4 août 1996 sur le bien-être des travailleurs lors de l'exécution de leur travail et arrêtés royaux du 27 mars 1998 relatifs à la Politique du bien-être des travailleurs lors de l'exécution de leur travail, au service interne pour la prévention et la protection au travail et aux services externes pour la prévention et la protection au travail (Moniteur belge, 31 mars 1998). 
des lieux de travail ainsi que les mesures prises par l'entreprise en matière d'environnement, pour ce qui concerne leur influence sur les points précités.

- La réorganisation de tous les acteurs de la prévention, et en particulier la rénovation des services de sécurité et de médecine du travail, laquelle réorganisation exige qu'ils s'appuient sur les cinq types d'expertise suivants : ergonomie, psychosociologie, hygiène du travail, sécurité ainsi que toxicologie et médecine du travail. Deux structures d'expertise, multidisciplinaires, apparaissent: l'une étant interne à l'entreprise (les services internes de prévention et de protection) et l'autre, externe (les services externes de prévention et de protection). Les compétences manquantes à la première doivent être comblées par affiliation à une structure de services externes. Enfin, dans chaque entreprise occupant en moyenne 50 travailleurs au moins, un ou plusieurs comités de prévention et de protection au travail (CPPT) doivent être constitués et se réunir une fois par mois. Le CPPT se compose, de façon paritaire, de représentants des travailleurs et de l'employeur. Il s'agit donc d'une structure de concertation sociale dont les tâches s'inscrivent dans la mise en œuvre de la politique de prévention. Celle-ci n'a de chance d'aboutir que si elle repose sur la concertation et une participation de toutes les composantes de l'entreprise. Or, le CPPT est idéalement placé pour assurer cette concertation puisque c'est au comité que les travailleurs confient ce qu'ils vivent sur le terrain et que c'est au cours de ses réunions que des informations sont échangées entre les différentes parties.

- L'obligation faite aux employeurs de mener une politique de prévention des risques qui repose sur une série de principes visant avant tout à éliminer les risques à la base. Ainsi, l'employeur est tenu de mettre en place un système dynamique de gestion des risques, une approche structurée et planifiée de la prévention, qui fait de l'analyse et de la gestion des risques le fil conducteur de la politique de l'entreprise en matière de bien-être. L'analyse des risques s'opère au niveau de l'organisation dans son ensemble, au niveau de chaque groupe de postes de travail ou de fonctions et au niveau individuel. La prévention ne doit pas uniquement porter sur les terrains classiques que sont la sécurité et la santé, mais également sur la charge psychosociale au travail (stress, épuisement professionnel, charge mentale et émotionnelle de travail, etc.), l'ergonomie et l'embellissement des lieux de travail. Toute cette politique de prévention doit être consignée dans deux documents : un plan global de prévention, d'une durée de cinq ans, et un plan d'action annuel.

Mentionnons enfin que la législation belge ne définit pas d'objectif opérationnel précis (ce qui est quasi impossible à définir, d'ailleurs) : elle prévoit la façon d'envisager le stress et de gérer ce risque, mais la marge de manœuvre et le seuil de bien-être à atteindre sont à définir dans chaque entreprise. Cela rend d'autant plus nécessaire la participation de tous les acteurs de l'entreprise.

Plus récemment, une convention collective de travail conclue au sein du Conseil national du travail - organe qui, précisons-le, est composé d'organisations interprofessionnelles d'employeurs et de travailleurs - porte plus particulièrement sur le stress en entreprise. Il s'agit de la CCT n 72 du 30 mars 1999, rendue obligatoire par un arrêté royal du 21 juin 1999 (Moniteur belge du 9 juillet 1999). On y donne la définition du stress suivante : « Le stress est un état perçu comme négatif par un groupe de travailleurs, qui s'accompagne de plaintes ou de 
dysfonctionnements au niveau physique, psychique et/ou social et qui est la conséquence du fait que des travailleurs ne sont pas en mesure de répondre aux exigences et attentes qui leur sont posées par leur situation de travail ». Le stress doit donc être conçu comme un phénomène collectif, caractéristique d'un ensemble de travailleurs, et non individuel, et sa source est à rechercher dans les conditions de travail. Dans le cadre de cette convention collective, l'employeur est tenu de détecter les risques éventuels de stress, d'en effectuer une évaluation sur la base de l'analyse de la situation de travail et de prendre, en fonction de cette évaluation, les mesures appropriées afin de prévenir les risques ou d'y remédier. Les mesures prises par l'employeur doivent donc être valables pour un ensemble de travailleurs, et non pour chaque travailleur individuellement.

Cette convention collective a réellement entraîné la mobilisation des entreprises dans la lutte contre les risques psychosociaux et le stress. Petit à petit, les entreprises se sont informées, se sont formées et ont réfléchi à un plan stratégique de prévention. Des accords intersectoriels ont également été signés, notamment dans le secteur des banques et de l'assurance. Cet accord s'est élargi au niveau européen en 2004 puisqu'un accord-cadre européen sur le stress professionnel ${ }^{3}$, proche dans ses principes de prévention de notre convention collective nationale, a été signé par la Confédération européenne des syndicats.

La restructuration de la législation a simultanément stimulé les programmes de recherche d'appui scientifique à la protection des travailleurs en matière de santé. En 1994, la Politique scientifique fédérale a ainsi favorisé la création d'instruments et de méthodes destinés à la surveillance du bien-être au travail. C'est ainsi que, dans le cadre du programme d'appui scientifique à la protection de la santé des travailleurs (contrats ST/12/011 de 1994 à 1998 et PS/12/04 de 1999 à 2000), le Service de psychologie du travail et des entreprises de l'Université de Liège (Hansez, 2001) a conçu le Working Conditions and Control Questionnaire (WOCCQ), un questionnaire destiné à évaluer le contrôle que les travailleurs ont de leurs conditions de travail. Le diagnostic complet comprend aussi une évaluation du stress professionnel positif et négatif et un relevé des situations problématiques permettant de récolter des données qualitatives sur des situations stressantes décrites par les travailleurs. Cet outil est applicable à toutes les entreprises (de 150 personnes minimum), quel que soit le secteur d'activité, et permet d'orienter la mise en place d'une politique de prévention du stress basée sur la réduction des risques psychosociaux. Les données récoltées à l'aide de ces questionnaires livrent un aperçu précis et détaillé des groupes à risques en matière de stress et du type d'intervention à conduire auprès de ces groupes. Ce diagnostic a connu et connaît toujours un succès important dans les entreprises grâce, notamment, à l'impulsion donnée par la mise en application de la législation et grâce également au soutien du Service public fédéral Emploi, Travail et Concertation sociale, en collaboration avec le Fonds social européen, pour la diffusion des outils scientifiques créés et validés. Une réelle politique de transfert de l'expertise scientifique vers les utilisateurs est effectivement primordiale pour promouvoir un outil adapté aux besoins des entreprises. Parmi les outils proposés, citons le Working Conditions and Control Questionnaire (WOCCQ) ${ }^{4}$ qui permet, à

3. Le texte de l'accord-cadre européen sur le stress professionnel, signé le 8 octobre2004 par la Confédération européenne des syndicats, peut être consulté à l'adresse suivante : http ://www.etuc.org/a/459.

4. Méthode de diagnostic collectif des risques psychosociaux (stress) liés au travail, mise au point dans le Service de Psychologie du Travail et des Entreprises de l'Université de Liège. Pour plus d'information, consulter le site Internet à l'adresse suivante : www.woccq.be. 
partir d'un encodage structuré, de générer automatiquement les analyses des données et les graphiques des résultats, la convention d'utilisation - incluant le respect de certaines règles déontologiques -, la formation à l'utilisation du WOCCQ et le recours à l'e-implementation (outil e-WOCCQ) pour la formation à distance des intervenants en entreprise.

Depuis une dizaine d'années, mais surtout après l'introduction de la nouvelle législation sur le bien-être en 1996, le Service de psychologie du travail a réalisé près de 90 études de diagnostic des risques psychosociaux en entreprise. Ce riche matériau stimule aujourd'hui l'investigation des difficultés pour les entreprises de passer du diagnostic des risques psychosociaux au plan d'action. Une étude exploratoire portant sur 24 entretiens avec des acteurs du bien-être dans des entreprises qui ont fait l'objet d'une étude de diagnostic met en évidence, parmi 36 facteurs proposés, les éléments qui facilitent ou bloquent le processus d'intervention en matière de risques psychosociaux. Parmi les facteurs facilitants, on trouve en tête le fait d'être mis au courant des résultats du diagnostic et le fait de transmettre ces résultats de manière appropriée. Apparaît seulement en troisième position la participation des travailleurs, facteur de succès des interventions souvent cité dans la littérature scientifique. Parmi les facteurs qui bloquent le processus, on trouve d'abord les changements organisationnels mais aussi le fait de focaliser les interventions sur un service en particulier au lieu de prendre en compte l'ensemble de l'entreprise. Privilégier une bonne communication des résultats, surtout dans un climat de changement, semble donc aussi important, sinon davantage, que la participation des travailleurs à la démarche. Le public-cible des interventions devrait aussi idéalement être défini après discussion avec les travailleurs.

\section{Loi sur la violence et le harcèlement moral ou sexuel au travail}

En Europe, un mouvement général de prise de conscience du phénomène de la violence et du harcèlement au travail s'est développé ces quinze dernières années. En Belgique, une étude sur les violences au travail (Garcia, 2001), met en évidence les chiffres suivants: $11,5 \%$ de harcèlement moral, $8 \%$ de harcèlement sexuel et 3,5\% de violence physique. Il est apparu indispensable de réagir face à ces comportements abusifs dont les conséquences psychologiques, physiques, sociales et même économiques se sont révélées innombrables (Service public fédéral Emploi, Travail et Concertation sociale, 2005).

Sur le plan législatif, les premières mesures datent de 1992, avec l'arrêté royal du 18 septembre organisant la protection des travailleurs contre le harcèlement sexuel sur les lieux de travail. Dans les années 1990, les premières recherches sur les dégâts du harcèlement moral sont publiées (Hirigoyen, 1998 ; Leymann, 1996 ; Vartia, 1996). En 1998, le harcèlement moral entre dans le code pénal et devient un délit. Il faudra attendre la législation du 11 juin 2002, son arrêté royal d'application et sa circulaire du 11 juillet 2002 pour qu'entre en vigueur une disposition légale portant sur le plan de la prévention et la prise en charge dans le lieu de travail (Ministère fédéral de l'Emploi et du Travail, 2002). La protection des travailleurs contre la violence et le harcèlement moral ou sexuel au travail a été ajoutée aux domaines qui font partie de la notion de bien-être dans la loi de 1996 sur le bien-être des travailleurs. Ainsi, les principes généraux sur lesquels la loi sur le bien-être s'appuie, tout comme ses arrêtés d'exécution, sont applicables à la protection contre la violence et le harcèlement moral ou sexuel au travail ; seules des dispositions particulières à ce domaine ont été ajoutées dans un chapitre (Service public fédéral Emploi, 
Travail et Concertation sociale, 2005). La législation définit le harcèlement moral comme «les conduites abusives et répétées de toute origine, externe ou interne à l'entreprise ou l'institution, qui se manifestent notamment par des comportements, des paroles, des intimidations, des actes, des gestes et des écrits unilatéraux, ayant pour objet ou pour effet de porter atteinte à la personnalité, la dignité ou l'intégrité physique ou psychique d'un travailleur (ou d'une autre personne à qui la loi s'applique) lors de l'exécution de son travail, de mettre en péril son emploi ou de créer un environnement intimidant, hostile, dégradant, humiliant ou offensant ».

La loi prévoit des dispositions particulières par rapport à la législation de 1996 sur le bien-être. Dans la perspective de la prévention des risques, ou prévention primaire, la loi prévoit tout d'abord la désignation du conseiller en prévention spécialisé dans les aspects psychosociaux du travail et des éventuelles personnes de confiance. Elle prévoit également l'aménagement matériel des lieux de travail, l'application des obligations imposées à la ligne hiérarchique ainsi que l'information et la formation des travailleurs comme des membres du CPPT. En ce qui concerne la prévention secondaire, visant à prévenir les dommages, la loi insiste sur la nécessité de déterminer les moyens nécessaires dont dispose la victime de harcèlement ou de violence pour obtenir de l'aide. Notamment, elle définit la manière de joindre le conseiller en prévention spécialisé ou la personne de confiance, ou encore la manière d'accueillir, d'aider et de soutenir les victimes. Il faut donc veiller à ce qu'une investigation ait lieu rapidement et en toute équité des faits qui se sont produits au travail. Au regard de la prévention tertiaire, la loi insiste, pour limiter les dommages, sur les mesures de prise en charge et de remise au travail des victimes. L'employeur doit veiller à ce que les travailleurs victimes de violence ou de harcèlement moral ou sexuel reçoivent le soutien psychologique approprié auprès des services ou des institutions spécialisés. Les missions du conseiller en prévention spécialisé, qui intervient dans le cadre de la politique de protection des victimes, sont également clairement définies dans la législation : aide, accueil, conseil et assistance à la victime, réception de la plainte motivée, communication de la plainte à l'employeur, examen de cette plainte, proposition de mesures à l'employeur pour mettre fin aux actes de violences ou de harcèlement ainsi que rapport collectif mais anonyme de faits de violence et de faits de harcèlement moral ou sexuel au travail.

Par ailleurs, la loi élargit le rôle de la personne de confiance tel qu'il a d'abord été défini dans l'arrêté royal du 18 septembre 1992 sur le harcèlement sexuel. Ce rôle, dont la désignation reste facultative, peut être confié à un collaborateur interne ou externe de l'entreprise et il n'impose aucune exigence en ce qui a trait à la formation et à l'expérience. Il consiste à assister le conseiller en prévention spécialisé, notamment dans l'élaboration des procédures, et à se concerter avec lui. Ce rôle comprend également l'accueil, l'aide, le conseil et l'assistance à la victime, notamment en réceptionnant une plainte motivée de la victime et en remettant cette plainte au conseiller en prévention spécialisé.

Plusieurs procédures sont possibles pour la victime afin de mettre fin aux comportements qu'elle juge abusifs à son égard. Elle peut privilégier la résolution interne, appelée procédure interne, en faisant appel aux services de la personne de confiance ou du conseiller en prévention spécialisé. Cela n'empêche cependant pas le travailleur de s'adresser directement à l'employeur ou à un membre de la ligne hiérarchique. La procédure interne prévoit que la personne de confiance ou le conseiller en prévention spécialisé peut, à la demande de la victime, tenter de concilier la victime et l'auteur des faits de violence ou de harcèlement. Lorsque la conciliation n'aboutit pas à un 
résultat ou paraît impossible, la personne de confiance actera la plainte motivée de la victime sur demande formelle de ladite victime. À partir du dépôt de cette plainte, la victime est protégée contre le licenciement et l'employeur est informé immédiatement par le conseiller en prévention spécialisé du fait que le travailleur jouit de cette protection. Deux autres voies d'action sont possibles pour la victime : elle peut également s'adresser aux fonctionnaires chargés de la surveillance, notamment les médecins-inspecteurs du travail ou elle peut intenter une procédure devant la juridiction compétente (Ministère fédéral de l'Emploi et du Travail, 2002).

\section{Quels enseignements tirer des trois années d'existence du cadre légal sur la violence et le harcèlement moral ou sexuel en Belgique ?}

Afin de faire un bilan de la situation trois ans après l'adoption de la loi sur la violence et le harcèlement au travail, le Service de psychologie sociale des groupes et des organisations de l'Université de Liège a mis en place un vaste programme de recherche-action qui comprend, entre autres, l'interview de professionnels s'occupant de harcèlement et de conflits au travail ainsi que leur mise en réseau par l'intermédiaire de groupes au sein desquels ils ont pu parler de leurs pratiques et expériences. Voyons ce qu'il ressort de ces diverses discussions.

Premièrement, la loi a fait apparaître une nouvelle responsabilité pour l'organisation : prévenir et prendre en charge les situations de violence et de harcèlement. Après son adoption, de nombreuses personnes ont demandé de l'aide, au point souvent de submerger de travail les professionnels chargés de cette mission.

Deuxièmement, il semble que, sous l'appellation de harcèlement moral au travail, les travailleurs ont réuni une multitude de difficultés qu'ils vivaient au travail. Pour l'essentiel, il s'agit de situations de souffrance relationnelle, que nous avons définies comme des situations dans lesquelles une personne - ou un groupe - exprime ou ressent un mal-être dû aux relations qu'elle vit sur son lieu du travail ou en rapport avec celui-ci (Delvaux et al., 2005). Parmi cellesci, on trouve plusieurs types d'hyperconflits (Faulx, Erpicum, Horion, 2005) et des situations dans lesquelles les personnes sont l'objet de comportements «déplacés » de la part de leurs collègues, sans pour autant que l'on puisse parler de harcèlement au sens de la loi.

Le fait que ces situations problématiques sur le plan relationnel aient été dénoncées sous l'étiquette de harcèlement moral au travail n'est pas sans poser problème. Premièrement, les professionnels ont dû faire face à des demandes d'intervention dans des situations de réelle souffrance, mais qui n'entrent pas nécessairement dans le cadre défini par la loi. Deuxièmement, la plupart des plaintes se sont vues opposer une fin de non-recevoir en raison d'un problème d'inadéquation entre les faits rapportés et le concept de harcèlement moral. Une certaine confusion en a résulté entre les personnes réclamant de l'aide et les professionnels chargés de les prendre en charge. Ainsi, mieux connaître l'ensemble des souffrances relationnelles au travail et pouvoir y répondre adéquatement constitue probablement le nouveau défi qui se présente aujourd'hui aux professionnels depuis la parution de la loi.

Troisièmement, il semble que la loi ait focalisé l'attention du public sur les démarches relatives à la plainte davantage que sur les actions préventives ou les règlements à l'amiable, qui constituent pourtant des voies privilégiées par le législateur. Nous avons relié ce phénomène à la 
prépondérance du modèle «dommage-plainte-réparation» (Faulx, Delvaux, Horion, 2005). Ainsi, la plainte apparaît souvent comme la seule réaction possible à un dommage et la réparation, comme seul moyen de compenser le dommage, avec pour conséquence que d'autres voies prévues dans la loi - comme la conciliation, par exemple -, sont négligées.

Quatrièmement, plusieurs signes laissent penser que l'on assiste à une évolution de l'image sociale du harcèlement moral au travail. Les travailleurs semblent de plus en plus suspicieux envers leurs collègues qui se plaignent de harcèlement et les plaignants, de moins en moins nombreux, tandis que plusieurs personnes estiment que le terme a été utilisé de manière abusive ou a été galvaudé. Après un effet médiatique spectaculaire qui a mis la thématique sur le devant de la scène, on assiste à une relativisation, que certains assimilent à une banalisation. De notre point de vue, cela pourrait avoir comme effet positif de recadrer le sens exact du terme harcèlement moral dans la mesure où d'autres catégories sémantiques seront proposées pour les autres formes de souffrance au travail, sans quoi les personnes continueront à appeler harcèlement l'ensemble des facteurs humains qui rendent leur vie au travail difficile.

Cinquièmement, l'écrasante majorité des affaires qui ont été traitées par la voie judiciaire n'ont pas donné raison au plaignant ou ont été classées sans suite. Il semble que le nombre de personnes qui se présentent au tribunal pour des affaires de harcèlement moral a fortement chuté depuis un an ou deux. On peut considérer ce phénomène comme positif - en convenant que les personnes savent mieux ce qu'est le harcèlement et qu'elles ont moins tendance à se plaindre de manière abusive -, ou comme négatif - en y voyant un signe de la perte de l'espoir qu'avait fait naître la loi chez tous ceux qui étaient victimes de harcèlement au travail. Nous n'avons pas assez de recul actuellement pour trancher.

Enfin, on peut signaler que la plupart des professionnels ont manifesté le besoin de réfléchir non seulement sur le harcèlement au sens strict, mais également sur l'ensemble des souffrances relationnelles au travail qui ont été mises en lumière ces dernières années.

\section{Bibliographie}

Delvaux S., Devacht I., Vandepoel M., Schrapen S., Faulx D. (2005). Concepts et phénomènes de souffrance relationnelle au travail, in Leroy J.F., Devacht I., Faulx D., Antonissen D. (éds), Agir sur les souffrances relationnelles au travail: Manuel de l'intervenant confronté aux situations de conflit, de harcèlement et d'emprise au travail, Bruxelles, Service public fédéral Emploi, Travail et Concertation sociale, 14-24.

Faulx D., Delvaux S., Horion E. (2005). Les bonnes pratiques transversales relatives aux situations de souffrance relationnelle au travail, in Leroy J.F., Devacht I., Faulx D., Antonissen D. (éds), Agir sur les souffrances relationnelles au travail: Manuel de l'intervenant confronté aux situations de conflit, de harcèlement et d'emprise au travail, Bruxelles, Service public fédéral Emploi, Travail et Concertation sociale, 220-236. 
Faulx D., Erpicum F., Horion E. (2005). Effet Gavroche et relations hyperconflictuelles de travail, Interactions, 9 (1) : 89-117.

Fondation européenne pour l'amélioration des conditions de vie et de travail (1997). Les contraintes de temps et l'autonomie au travail dans l'Union européenne, Luxembourg, Office des publications officielles des communautés européennes, 8 .

Garcia A. (2001). Le harcèlement dans les lieux de travail : caractéristiques et conséquences du harcèlement moral sur les travailleurs masculins et féminins, Bruxelles, Service public fédéral Emploi, Travail et Concertation sociale (avec le soutien du Fonds social européen), 333.

Hansez I. (2001). La validation du WOCCQ : vers un modèle transactionnel du stress et du contrôle de l'activité de travail, thèse de doctorat en psychologie non publiée, Liège, Université de Liège.

Hirigoyen M.F. (1998). Le harcèlement moral : la violence au quotidien, Paris, Syros, 213.

Leymann H. (1996). The content and development of mobbing at work, European Journal of Work and Organizational Psychology, 5 (2) : 165-184.

Ministère fédéral de l'Emploi et du Travail (2000). Le bien-être des travailleurs lors de l'exécution de leur travail : Commentaire juridique de la loi du 4 août 1996, Bruxelles, Le Ministère, 68.

Ministère fédéral de l'Emploi et du Travail (2002). La protection contre la violence, le harcèlement moral ou sexuel au travail : Commentaire juridique de la loi du 11 juin 2002, Bruxelles, Le Service, 66.

Sauter S.L., Murphy L.R. (1995). The changing face of work and stress, in Sauter S.L., Murphy L.R. (éds.), Organizational risk factors for job stress, Washington, American Psychological Association, 1-6.

Service public fédéral Emploi, Travail et Concertation sociale (2005). Clés pour... Prévenir et lutter contre la violence et le harcèlement moral ou sexuel au travail, Bruxelles, Le Service, 33.

Vartia M. (1996). The sources of bullying: Psychological work environment and organizational climate, European Journal of Work and Organizational Psychology, 5 (2) : 203-214. 